\title{
KERNELS OF REPRESENTATIONS AND COIDEAL SUBALGEBRAS OF HOPF ALGEBRAS
}

\author{
SEBASTIAN BURCIU \\ Faculty of Mathematics and Computer Science, University of Bucharest, \\ 14 Academiei Street, Bucharest, Romania and \\ Institute of Mathematics "Simion Stoilow" of the Romanian Academy, P.O. Box 1-764, \\ Bucharest, 014700, Romania \\ e-mail: sebastian.burciu@imar.ro
}

(Received 14 December 2010; accepted 4 May 2011; first published online 2 August 2011)

\begin{abstract}
We define left and right kernels of representations of Hopf algebras. In the case of group algebras, left and right kernels coincide and they are the usual kernels of modules. In the general case, we show that these kernels coincide with the categorical left and right Hopf kernels of morphisms of Hopf algebras defined in Andruskiewitsch and Devoto [Extensions of Hopf algebras, Algebra i Analiz 7 (1995), 22-69]. Brauer's theorem for kernels over group algebras is generalised to Hopf algebras.
\end{abstract}

2010 Mathematics Subject Classification. MSC 16W30

1. Introduction. If $G$ is a finite group and $M$ is a representation of $G$ which affords the character $\chi$, then the kernel of the representation $M$ is defined as the set of all $g \in G$ which act as the identity on $M$. It is easy to see that $\operatorname{ker}_{G}(\chi)$ is a normal subgroup of $G$. The notion of kernel of a representation of a group is a very important notion in studying the representations of groups (see [9]). For example, a classical result of Brauer in group theory states that over an algebraically closed field $k$ of characteristic zero, if $\chi$ is a faithful character of $G$, then any other irreducible character of $G$ is a direct summand in some tensor power of $\chi$.

In [5], the author introduced a similar notion for the kernel of representations of any semisimple Hopf algebra. The notion uses the fact that the exponent of a semisimple Hopf algebra is finite [7]. In this paper, we will extend the notion of kernel to arbitrary Hopf algebras, not necessarily semisimple. The aforementioned Brauer's theorem for groups was extended in [5] for semisimple Hopf algebras $A$, and for those representations of $A$ whose characters are central in $A^{*}$. With the help of this new notion of kernel, we extend this theorem to an arbitrary representation whose character is not necessarily central in the dual Hopf algebra (see Theorem 4.2.1). We also prove a version of Brauer's theorem for arbitrary Hopf algebras, giving a new insight to the main results from [12] and [13].

Note that the group algebra $k G$ is cocommutative. Lack of cocommutativity suggests the introduction of left and right kernels of modules. It will also be shown that these left and right kernels coincide with the left and, respectively, right categorical kernels of morphisms of Hopf algebras that has been introduced in [2] and extensively studied in [1]. This coincidence proven in Theorem 2.3.6 suggests also how to generalise the notion of kernel of modules to the non-semisimple case. 
Recently, it was proven in [4] that Hopf subalgebras are normal if and only if they are depth two subalgebras. We extend this result to coideal subalgebras. Moreover, we show that in this situation, depth two and normality in the sense defined by Rieffel in [14] also coincide. Normal subalgebras as defined by Rieffel were recently revised in Section 4 of [6].

The paper is organised as follows. Section 2 introduces the notion of left and right kernels of modules and proves their coincidence with the categorical kernels introduced in [2]. A general version of Brauer's theorem is also stated in this section. Section 3 is concerned with depth two coideal subalgebras of a Hopf algebra. We prove a left coideal subalgebra is right depth two subalgebra if and only if it is a left normal coideal subalgebra. Section 4 considers coideal subalgebras of semisimple Hopf algebra. An extension of Brauer's theorem in this case is also considered.

The Hopf algebra notations from [11] are used in this paper but we drop the sigma symbol from Sweedler's notation of comultiplication. Recall a left coideal subalgebra $S$ of $A$ is a subalgebra of $A$ with $\Delta(S) \subset A \otimes S$. We say that a left coideal subalgebra $S$ of $A$ is left normal if and only if it is invariant under the left the adjoint action of $A$, i.e. $a_{1} x S\left(a_{2}\right) \in S$ for all $a \in A$ and $x \in S$. Such a coideal subalgebra will be called a left normal coideal subalgebra. Also, it follows from [15] that if $A$ is finite dimensional then $A$ is free as left or right $S$-module.

For a subalgebra $B$ of a Hopf algebra $A$, we denote by $B^{+}$the ideal of $B$ defined by $\omega(B)=B^{+}=A^{+} \cap B$. As usual, $A^{+}=\operatorname{ker} \epsilon$ is the kernel of the count $\epsilon$ of $A$.

2. Left and right kernels. Let $A$ be an arbitrary Hopf algebra over a field $k$ and $M$ be an $A$-module. We say that an element $a \in A$ acts trivially on $M$ if and only $a m=\epsilon(a) m$ for all $a \in A$. If $S$ is a subalgebra of $A$ we say that $S$ acts trivially on $M$ if each element of $S$ acts trivially on $M$.

If $M$ is an $A$-module then define

$$
\mathcal{A}_{\mathbf{M}}=\{a \in A \mid a m=\epsilon(a) m \text { for all } m \in M\} .
$$

It is easy to verify that $\mathcal{S}_{M}$ is a subalgebra of $A$, thus the largest subalgebra of $A$ which acts trivially on $M$. If $A$ is semisimple and $M$ a simple $A$-module then $\operatorname{dim}_{k}\left(\mathcal{S}_{M}\right)=\operatorname{dim}_{k}(A)-\operatorname{dim}_{k}(M)^{2}$.

2.1. Definition of left kernels. Let $M$ be an $A$-module and $\mathrm{L}_{M}: A \otimes M \rightarrow A \otimes M$ be the linear operator given by $a \otimes m \mapsto a_{1} \otimes a_{2} m$. Let $\operatorname{LKer}_{M}$ be the largest subspace $B$ of $A$ such that $\left.\mathrm{L}_{M}\right|_{B \otimes M}=\mathrm{id}$. Then $\operatorname{LKer}_{M}$ is called the left kernel of $M$. Thus

$$
\operatorname{LKer}_{M}=\left\{a \in A \mid a_{1} \otimes a_{2} m=a \otimes m, \text { for all } m \in M\right\} .
$$

If $A$ is a semisimple Hopf algebra and $M$ a module with character $\chi$ we also write $\mathrm{LKer}_{\chi}$ instead of $\mathrm{LKer}_{M}$.

REMARK 2.1.2. If $A=k G$ and $M$ a $G$-module then $\operatorname{LKer}_{M}$ is the group algebra $k\left[\operatorname{Ker}_{G}(M)\right]$ of the usual kernel of $M$.

Proposition 2.1.3. Let $A$ be a Hopf algebra and $M$ be an A-module. Then $\operatorname{LKer}_{M}$ is a left normal coideal subalgebra of $A$. 
Proof. Applying $\epsilon \otimes \mathrm{Id}$ to the defining relation 2.1.1 of $\operatorname{LKer}_{M}$ it follows that $\mathrm{LKer}_{M} \subset \mathcal{S}_{M}$. It is easy to verify that $\mathrm{LKer}_{M}$ is an algebra.

Next, we will show that $\Delta\left(\operatorname{LKer}_{M}\right) \subset A \otimes \operatorname{LKer}_{M}$. Let $a \in \operatorname{LKer}_{M}$ and $\Delta(a)=$ $\sum_{i=1}^{n} h_{i} \otimes x_{i}$, where $h_{i}$ is a basis of $A$. It will be shown that $x_{i} \in \operatorname{LKer}_{M}$ for all $1 \leq i \leq n$. Indeed, $\Delta^{2}(a)=\sum_{i=1}^{n} h_{i} \otimes \Delta\left(x_{i}\right)$. Since $a_{1} \otimes a_{2} m=a \otimes m$, it follows that $a_{1} \otimes a_{2} \otimes$ $a_{3} m=a_{1} \otimes a_{2} \otimes m$ for all $m \in M$. Thus $\sum_{i=1}^{n} h_{i} \otimes\left(x_{i}\right)_{1} \otimes\left(x_{i}\right)_{2} m=\sum_{i=1}^{n} h_{i} \otimes x_{i} \otimes m$ which implies that $\left(x_{i}\right)_{1} \otimes\left(x_{i}\right)_{2} m=x_{i} \otimes m$ for all $m \in M$ and $1 \leq i \leq n$.

Now, we will show invariance under the left adjoint action of $A$. Let $h \in A$ and $a \in \operatorname{LKer}_{M}$. Then $h_{1} a S\left(h_{2}\right) \in \operatorname{LKer}_{M}$ since

$$
\begin{aligned}
\left(h_{1} a S\left(h_{2}\right)\right)_{1} \otimes\left(h_{1} a S\left(h_{2}\right)\right)_{2} m & =h_{1} a_{1} S\left(h_{4}\right) \otimes h_{2} a_{2} S\left(h_{3}\right) m \\
& =h_{1} a S\left(h_{4}\right) \otimes h_{2} S\left(h_{3}\right) m \\
& =h_{1} a S\left(h_{2}\right) \otimes m .
\end{aligned}
$$

Proposition 2.1.4. Let $A$ be a Hopf algebra and

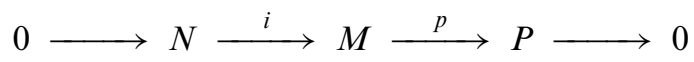

be a short exact sequence of modules. Then

$$
\operatorname{LKer}_{M} \subseteq \operatorname{LKer}_{N} \cap \operatorname{LKer}_{P} .
$$

Proof. Clearly, $\operatorname{LKer}_{M} \subset \operatorname{LKer}_{N}$. Next, we will show $\operatorname{LKer}_{M} \subset \operatorname{LKer}_{P}$. Indeed, if $a \in \operatorname{LKer}_{M}$ then $a_{1} \otimes a_{2} m=a \otimes m$ for all $m \in M$ and applying id $\otimes p$ it follows that $a \in \operatorname{LKer}_{P}$ since $p$ is surjective.

Recall that the exponent of $A$ is the smallest positive number $m>0$ such that $a^{[m]}=\epsilon(a) 1$ for all $a \in A$. The generalised power $a^{[m]}$ is defined by $a^{[m]}=a_{1} a_{2} \ldots a_{m}$.

Remark 2.1.5. Let $A$ be a Hopf algebra and $M$ be an $A$-module. Then it is easy to check that $L_{M}^{s}(a \otimes m)=a_{1} \otimes a_{2}^{[s]} m$ for all $s \geq 1$. Thus if $A$ is of a finite exponent $m$ then $L_{M}^{m}=$ id on $A \otimes M$.

THEOREM 2.1.6. Let $A$ be a Hopf algebra of finite exponent over an algebraically closed field $k$ and let

$$
0 \longrightarrow N \stackrel{i}{\longrightarrow} M \stackrel{p}{\longrightarrow} P \longrightarrow 0
$$

be a short exact sequence of finite-dimensional modules. Then

$$
\operatorname{LKer}_{M}=\operatorname{LKer}_{N} \cap \operatorname{LKer}_{P} .
$$

Proof. Let $L:=\operatorname{LKer}_{N} \cap \operatorname{LKer}_{P}$ and consider the exact sequence

$$
0 \longrightarrow L \otimes N \stackrel{1 \otimes i}{\longrightarrow} L \otimes M \stackrel{1 \otimes p}{\longrightarrow} L \otimes P \longrightarrow 0
$$

If $l \in L$ and $m \in M$ then

$(1 \otimes p)\left(L_{M}(l \otimes m)-l \otimes m\right)=l_{1} \otimes p\left(l_{2} m\right)-l \otimes p(m)=l_{1} \otimes l_{2} p(m)-l \otimes p(m)=0$ 
since $l \in \operatorname{LKer}_{P}$. Thus $L_{M}(l \otimes m)-l \otimes m \in L \otimes N$. It follows that $L_{N}\left(L_{M}(l \otimes m)-\right.$ $l \otimes m))=L_{M}(l \otimes m)-l \otimes m$. This also implies that $L_{M}^{2}(l \otimes m)-L_{M}(l \otimes m)=L_{M}(l \otimes$ $m)-l \otimes m$. Thus $\left(L_{M}-\mathrm{id}\right)^{2}=0$ on $L \otimes M$. On the other hand, since $A$ has finite exponent, one has $L_{M}^{m}=$ id on $L \otimes M$ by the previous remark. Since $k$ is algebraically closed, it follows that $L_{M}=$ id on $L \otimes M$, i.e. $L \subset \operatorname{LKer}_{M}$.

REMARK 2.1.7. Among the Hopf algebras with finite exponent we recall semisimple algebras and group algebras of finite groups over a field of characteristic diving the order of the group. The fact that the exponent of a finite-dimensional semisimple Hopf algebra is finite was proven in [7]. In the same paper, it is also shown that in this case the exponent divides the third power of the dimension of $A$.

REMARK 2.1.8. Let $S_{M}$ the inverse operator of $L_{M}$. Then $S_{M}$ is given by $S_{M}(a \otimes m)=$ $a_{1} \otimes S\left(a_{2}\right) m$ for all $a \in A$ and $m \in M$. It follows that $\left.S_{M}\right|_{\text {LKer }_{M} \otimes A}=$ id and thus one also has that

$$
\operatorname{LKer}_{M}=\left\{a \in A \mid a_{1} \otimes S\left(a_{2}\right) m=a \otimes m, \text { for all } m \in M\right\} .
$$

Proposition 2.1.9. Let $A$ be a Hopf algebra and $M$ be an A-module. Then $\operatorname{LKer}_{M}$ is the largest coideal subalgebra of $A$ that acts trivially on $M$.

Proof. Proposition 2.1.3 implies that $\mathrm{LKer}_{M}$ acts trivially on $M$. On the other hand, if $\Delta(S) \subset A \otimes S$ and $S$ acts trivially on $M$ then $s_{1} \otimes s_{2} m=s_{1} \otimes \epsilon\left(s_{2}\right) m=s \otimes m$, which shows that $S \subset \mathrm{LKer}_{M}$.

Lemma 2.1.10. Let $A$ be a Hopf algebra with bijective antipode and $M$ be a finitedimensional module over A. Then $\operatorname{LKer}_{M}=\operatorname{LKer}_{M^{*}}$.

Proof. For the operator $L_{M^{*}}: A \otimes M^{*} \rightarrow A \otimes M^{*}$ one has that $\operatorname{LKer}_{M^{*}}=\{a \in$ $\left.A \mid a_{1} \otimes a_{2} f=a \otimes f\right\}$ for all $f \in M^{*}$. This is equivalent to $a_{1} f\left(S\left(a_{2}\right) m\right)=a f(m)$ for all $m \in M$ and $f \in M^{*}$. Therefore, $a \in \operatorname{LKer}_{M^{*}}$ if and only if $a_{1} \otimes S\left(a_{2}\right) m=a \otimes m$ for all $m \in M$ which implies by Remark 2.1.8 that $\operatorname{LKer}_{M^{*}}=\operatorname{LKer}_{M}$.

2.2. Definition of right kernel. Let $M$ be an $A$-module and $\mathrm{R}_{M}: A \otimes M \rightarrow$ $A \otimes M$ given by $a \otimes m \mapsto a_{2} \otimes a_{1} m$. Let $\operatorname{RKer}_{M}$ be the largest subspace $B$ of $A$ such that $\left.\mathrm{R}_{M}\right|_{B \otimes M}=\mathrm{id}$. Thus

$$
\operatorname{RKer}_{M}=\left\{a \in A \mid a_{2} \otimes a_{1} m=a \otimes m, \text { for all } m \in M\right\} .
$$

Suppose that $A$ has a bijective antipode $S$ with inverse $S^{-1}$. Then the inverse operator $U_{M}$ of $\mathrm{R}_{M}$ is given by $U_{M}(a \otimes m)=a_{2} \otimes S^{-1}\left(a_{1}\right) m$ for all $a \in A$ and $m \in M$. It follows that $\left.U_{M}\right|_{\operatorname{RKer}_{M} \otimes A}=$ id and thus one has also that

$$
\operatorname{RKer}_{M}=\left\{a \in A \mid a_{2} \otimes S^{-1}\left(a_{1}\right) m=a \otimes m, \text { for all } m \in M\right\} .
$$

It is also easy to check that $\Delta\left(\mathrm{RKer}_{M}\right) \subset \mathrm{RKer}_{M} \otimes A$.

REMARK 2.2.3. (1) Suppose that the Hopf algebra $A$ has a bijective antipode and let $M$ be an $A$-module. Then $\operatorname{RKer}_{M}=S\left(\operatorname{LKer}_{M}\right)$. Indeed, if $a \in \operatorname{LKer}_{M}$ then

$$
S(a)_{2} \otimes S(a)_{1} m=S\left(a_{1}\right) \otimes S\left(a_{2}\right) m=S(a) \otimes m,
$$


which shows that $S\left(\operatorname{LKer}_{M}\right) \subset \operatorname{RKer}_{M}$. Similarly, it can be checked that if $a \in \operatorname{RKer}_{M}$ then $S^{-1} a \in \operatorname{LKer}_{M}$. Thus $\operatorname{RKer}_{M}=S\left(\operatorname{LKer}_{M}\right)$.

(2) Applying $\epsilon \otimes \mathrm{Id}$ to the relation 2.2.1 it also follows that $\operatorname{RKer}_{M} \subset \mathcal{S}_{M}$.

\subsection{Description as categorical kernels.}

LemmA 2.3.1. If $M$ and $N$ are two A-modules then $\operatorname{LKer}_{M} \cap \operatorname{LKer}_{N} \subset \operatorname{LKer}_{M \otimes N}$. In particular, $\mathrm{LKer}_{M} \subset \mathrm{LKer}_{M^{\otimes n}}$ for all $n \geq 1$.

Proof. Suppose $m \in M, n \in M$ and $a \in \operatorname{LKer}_{M} \cap \operatorname{LKer}_{N}$. Since $a_{1} \otimes a_{2} n=a \otimes n$, applying $\Delta \otimes$ Id one has $a_{1} \otimes a_{2} \otimes a_{3} n=a_{1} \otimes a_{2} \otimes n$. Thus $a_{1} \otimes a_{2} m \otimes a_{3} n=a_{1} \otimes$ $a_{2} m \otimes n=a_{1} \otimes m \otimes n$.

Definition 2.3.2. Let $A$ be a Hopf algebra and $M$ be an $A$-module. We define the Hopf kernel $A_{M}$ of $M$ as the largest sub-bialgebra of $A$ contained in $\mathcal{S}_{M}$.

If $A$ is finite dimensional then $A_{M}$ is also the largest Hopf subalgebra of $A$. It is easy to see that in the case of a semisimple Hopf algebra this notion of kernel coincides with the kernel $A_{M}$ of the module $M$ introduced in [5].

Lemma 2.3.3. Let $A$ be a Hopf algebra and $M$ be an A-module. Then

(1) The kernel $A_{M}$ is the largest subcoalgebra in $\mathcal{S}_{M}$.

(2) The kernel $A_{M}$ is the largest subcoalgebra in $\mathrm{LKer}_{M}$.

(3) The kernel $A_{M}$ is the largest subcoalgebra in $\mathrm{RKer}_{M}$.

Proof.

(1) If $C$ is a subcoalgebra of $A$ contained in $\mathcal{S}_{M}$ then $<C>=\oplus_{n \geq 0} C^{n}$ is a subbialgebra of $A$. Thus one has $\left\langle C>\subset A_{M}\right.$.

(2) Clearly, $A_{M}$ is a subcoalgebra of $\operatorname{LKer}_{M} \subset \mathcal{S}_{M}$. On the other hand, any subcoalgebra of $\mathcal{S}_{M}$ is by definition included in $\operatorname{LKer}_{M}$. Thus the largest subcoalgebra of $\mathcal{S}_{M}$ is $A_{M}$ and coincides with the largest subcoalgebra of $\operatorname{LKer}_{M}$.

(3) The proof of (3) is similar to that of (2).

Proposition 2.3.4. Let $A$ be a Hopf algebra and $M$ be an A-module. Then

$$
A_{M}=\left\{a \in A \mid a_{1} \otimes a_{2} m \otimes a_{3}=a_{1} \otimes m \otimes a_{2} \text { for all } m \in M\right\} .
$$

Proof. Let $A_{M}^{\prime}=\left\{a \in A \mid a_{1} \otimes a_{2} m \otimes a_{3}=a_{1} \otimes m \otimes a_{2}\right.$ for all $\left.m \in M\right\}$. Clearly, $A_{M} \subset A_{M}^{\prime}$, since $A_{M}$ is a coalgebra. On the other hand, it easy to check that $A_{M}^{\prime}$ is a sub-bialgebra of $A$ contained inside $\mathcal{S}_{\mathrm{M}}$. Maximality of the kernel $A_{M}$ implies the other inclusion.

Let $\pi: A \rightarrow B$ a Hopf map. Recall that the Hopf kernel of $\pi$ was defined in [2] as

$$
\operatorname{HKer}(\pi)=\left\{a \in A \mid a_{1} \otimes \pi\left(a_{2}\right) \otimes a_{3}=a_{1} \otimes \pi(1) \otimes a_{2}\right\}
$$

Also, the left and right kernels of $\pi$ are defined as $\operatorname{LKer}(\pi)=A^{c o \pi}$ and $\operatorname{RKer}(\pi)={ }^{c o} \pi$.

Now, let $M$ be an $A$-module and $I_{M}=\cap_{n \geq 0} \operatorname{Ann}_{A}\left(M^{\otimes n}\right)$. Then $I_{M}$ is a Hopf ideal [12] that will be called the Hopf ideal generated by $M$ for the rest of the paper. Also, let $\pi_{M}: A \rightarrow A / I_{M}$ be the canonical projection. 
THEOREM 2.3.6. Suppose that $M$ is a finite-dimensional module over a finitedimensional Hopf algebra A. Let $I_{M}=\cap_{n \geq 0} \operatorname{Ann}_{A}\left(M^{\otimes n}\right)$ and $\pi: A \rightarrow A / I_{M}$ be the canonical projection. Then

(1) $A^{\text {co } \pi}=\operatorname{LKer}_{M}$ and ${ }^{\text {co } \pi} A=\mathrm{RKer}_{M}$.

(2) $\operatorname{Hker}(\pi)=A_{M}$.

Proof.

(1) If $a \in A^{c o \pi}$ then $a_{1} \otimes a_{2} m=a_{1} \otimes \pi\left(a_{2}\right) m=a \otimes \pi(1) m=a \otimes m$, therefore $a \in$ $\operatorname{LKer}_{M}$. Conversely, suppose that $a \in \operatorname{LKer}_{M}$ and let $\Delta(a)=\sum_{i=1}^{s} a_{i} \otimes x_{i}$ with $a_{i}$ a $k$-basis of $A$. Then $x_{i} \in \operatorname{LKer}_{M}$ and the Lemma 2.3.1 implies that $x_{i} \in$ LKer $_{M^{\otimes n}}$ for all $n \geq 1$. Therefore, $x_{i}-\epsilon\left(x_{i}\right) 1 \in I_{M}$ which implies that $\pi\left(x_{i}\right)=$ $\epsilon\left(x_{i}\right) 1$. Thus $A^{c o \pi}=\operatorname{LKer}_{M}$ and applying the antipode $S$ it follows that ${ }^{c o \pi} A=$ $\mathrm{RKer}_{M}$.

(2) It is easy to see that $A_{M} \subset \operatorname{HKer}(\pi)$. On the other hand, since $\operatorname{HKer}(\pi)$ acts trivially on $M$ it follows that $\operatorname{HKer}(\pi) \subset A_{M}$ by maximality of $A_{M}$.

The following corollary can be regarded as a generalisation of Brauer's theorem for groups.

Corollary 2.3.7. Suppose that $M$ is a finite-dimensional module over a finitedimensional Hopf algebra A. Then

$$
\cap_{n \geq 0} \operatorname{Ann}_{A}\left(M^{\otimes n}\right)=\omega\left(\operatorname{LKer}_{M}\right) A .
$$

For a coideal subalgebra $S$ of $A$ denote by $\epsilon_{S}$ the character of the left trivial $S$-module. Then $\epsilon_{S}$ is the restriction of the counit $\epsilon$ to $S$.

Remark 2.3.8. One has that $A \otimes_{S} k$, the trivial left $S$-module induced to $A$, is isomorphic to $A / A S^{+}$via the map $a \otimes_{S} 1 \mapsto \bar{a}$.

The next lemma is the first item of Theorem 1.1 of [16].

LeMma 2.3.9. Suppose that the antipode $S$ of the Hopf algebra $A$ is bijective. Let $S$ be a coideal subalgebra of $A$ and $\pi: A \rightarrow A / A S^{+}$the canonical coalgebra projection. If $A$ is left $S$-faithfully flat then $A^{\text {co } \pi}=S$.

REMARK 2.3.10. From Lemma 4.2 of [16] it follows that $S$ is normal whenever $A S^{+} \subset S^{+} A$.

Proposition 2.3.11. Let $L$ be a normal left coideal subalgebra of a Hopf algebra A with bijective antipode $S$. Then

$$
\operatorname{LKer}_{\epsilon_{L} \uparrow_{L}^{A}}=L
$$

Proof. We show first that $L \subset \operatorname{LKer}_{\epsilon_{L} \uparrow_{L}^{A}}$. Indeed, for all $l \in L$ and $a \in A$ one has

$$
\left.l_{1} \otimes l_{2}\left(S a \otimes_{L} 1\right)=l_{1} \otimes S a_{1} \otimes_{L}\left(a_{2} l_{2} S a_{3}\right) 1\right)=l \otimes\left(S a \otimes_{L} 1\right)
$$

since $L$ is normal. Since the antipode $S$ is bijective, it follows that $L \subset \operatorname{LKer}_{\epsilon_{L} \uparrow L}$. 
Let $\pi: A \rightarrow A / / L$ be the canonical projection. It follows that $A^{c o} \pi=L$ by Lemma 2.3.9. Suppose now that $a \in \operatorname{LKer}_{M}$ with $M=k_{L} \uparrow_{L}^{A} \cong A / A L^{+}$. Then $a \otimes m=a_{1} \otimes$ $a_{2} m=a_{1} \otimes \pi\left(a_{2}\right) m$ for all $m \in M$. In particular, for $m=\overline{1}$ one has $a_{1} \otimes \pi\left(a_{2}\right)=a \otimes$ $\pi(1)$. Therefore, $a \in A^{c o \pi}=L$.

CoROllary 2.3.13. Suppose that $L$ is a normal coideal subalgebra of $A$ and $M:=$ $k \uparrow_{L}^{A}$ is the trivial L-module induced up to A. Let $I_{M}$ be the Hopf ideal generated by $M$ in $A$ and $\pi: A \rightarrow A / I_{M}$ be the canonical projection. Then $A^{\text {co } \pi}=L$, i.e. $A / I_{M}=A / / L$.

Proof. By Proposition 2.3.11 it follows that $\operatorname{LKer}_{M}=L$. On the other hand, Corollary 2.3.7 implies that $A^{c o \pi}=\mathrm{LKer}_{M}$.

Next, we will give two examples of left (right) kernels.

EXAMPLE 2.3.14.

(1) Let $A$ be a Hopf algebra and consider the left adjoint action of $A$ on itself. Then $L:=\operatorname{LKer}(A)$ is the largest central coideal subalgebra of $A$. In this situation, the kernel $A_{A}$ of the adjoint action coincides with the largest central sub-bialgebra of $A$ and it is in fact a Hopf subalgebra called, the Hopf centre of $A$ in [1].

(2) Let $A$ be a finite-dimensional Hopf algebra and

$$
L:=\cap_{S \in \operatorname{Irr}(A)} \operatorname{LKer}(S) .
$$

Then $A / / L$ is the largest semisimple Hopf algebra quotient of $A$. Moreover, $A$ has Chevalley property if and only if the Jacobson radical $\operatorname{rad}(A)$ equals $\omega(L) A$.

Remark 2.3.15. Let $\pi: A \rightarrow H$ be a Hopf algebra map with $A$ and $H$ finitedimensional Hopf algebras. Then $H$ can be regarded as $A$-module via $\pi$ and let $L:=\operatorname{LKer}_{A}(H)$. It is to see that in this case $A / / L$ is isomorphic with the Hopf image of $\pi$ as defined in [3]. Thus $\pi$ is inner faithful if and only if $L$ is trivial.

2.4. Core of a coideal subalgebra. Let $S$ be a left coideal subalgebra of $A$. If $L$ and $K$ are left normal coideal subalgebras of $A$ contained in $S$ then it is easy to see that $L K$ is also a left normal coideal subalgebra contained in $S$. Thus one can define $L:=\operatorname{core}(S)$ as the largest left normal coideal subalgebra of $A$ contained in $S$.

The next proposition gives a description of the core of a coideal subalgebra. It generalises Theorem 3.7 and Remark 3.8 from [5].

Proposition 2.4.1. Suppose that $S$ is a coideal subalgebra of $A$ and let $L:=$ $\operatorname{LKer}_{\epsilon_{S} \uparrow_{S}^{A}}$. Then $\operatorname{core}(S)=L$.

Proof. First we show that $L \subset S$. Since $k \uparrow_{S}^{A}=A / A S^{+}$one has that

$$
L=\left\{l \in A \mid l_{1} \otimes \pi\left(l_{2} a\right)=l \otimes \pi(a) \text { for all } a \in A\right\}
$$

Thus for $a=1$ one gets that $L \subset A^{c o \pi}=S$. Now suppose that $K$ is any left normal coideal subalgebra of $A$ contained in $S$. Then one has a canonical projection of $A$ modules $A / A K^{+} \rightarrow A / A S^{+}$and Proposition 2.1.4 implies $K=$ $\mathrm{LKer}_{A / A K^{+}} \subset L$.

COROLlary 2.4.3. Let $S$ be a coideal subalgebra of $A$. Then $S$ is normal if and only if $\epsilon_{S} \uparrow_{S}^{A} \downarrow{ }_{S}^{A}=\frac{|A|}{|S|} \epsilon_{S}$ 
Proof. If $S$ normal the statement follows from Proposition 2.3.11. The converse follows from the previous proposition and Proposition 2.1.9.

2.5. On two endofunctors on $A$-mod and respectively $S$-mod. Let $A$ be a Hopf algebra. Then $A$ is a right comodule subalgebra of $A$ with the usual multiplication and comultiplication given by $\Delta$.

Let $S$ be a left $A$-comodule subalgebra of $A$, i.e. a left coideal subalgebra of $A$. Then for any $A$-module $M$ and any $S$-module $V$ the comodule structure $\rho: S \rightarrow A \otimes S$ defines via pullback, an $S$-module structure on $M \otimes V$. Denote this module structure by $M \odot V$.

This makes the category of $S$-modules a left module category over the tensor category $A$-modules.

Proposition 2.5.1. Let $S \subset A$ be a right $A$-comodule subalgebra of $A$. Then $M \otimes$ $V \uparrow{ }_{S}^{A} \cong\left(M \downarrow_{S}^{A} \odot V\right) \uparrow_{S}^{A}$ for any $S$-module $V$ and any $A$-module $M$.

Proof. The map $T: A \otimes_{S}(M \odot V) \rightarrow M \otimes\left(A \otimes_{S} V\right)$ given by $a \otimes_{S}(m \otimes v) \mapsto$ $a_{1} \otimes a_{1} m \otimes\left(a_{2} \otimes_{S} v\right)$ is a well-defined map and a morphism of $A$-modules with inverse given by $m \otimes\left(a \otimes_{S} v\right) \mapsto a_{2} \otimes_{S}\left(S^{-1}\left(a_{1}\right) m \otimes v\right)$.

COROllary 2.5.2. Let $S \subset A$ be a right $A$-comodule subalgebra of $A$. Then

$$
M \otimes \epsilon_{S} \uparrow \stackrel{A}{S}=M \downarrow_{S}^{A} \uparrow_{S}^{A}
$$

for any A-module $S$.

Proof. Put $V=k$, the trivial $S$-module in the above relation. Note also that $M \odot k=M \downarrow_{S}^{A}$.

Define the endofunctors:

$$
\mathcal{T}: S \text {-mod } \rightarrow S \text {-mod given by } \mathcal{T}(V)=V \uparrow_{S}^{A} \downarrow{ }_{S}^{A}
$$

for any $S$-module $V$. Also define

$$
\mathcal{V}: A \text {-mod } \rightarrow A \text {-mod given by } \mathcal{V}(M)=M \downarrow_{S}^{A} \uparrow{ }_{S}^{A}
$$

for any $A$-module $M$.

The following lemma is straightforward. It shows that the restriction functor res : $A$-mod $\rightarrow S$-mod is a morphism of $A$-mod categories.

Lemma 2.5.6. Let $S$ be a coideal subalgebra of $A$ and $M, N$ be two A-modules. Then

$$
(M \otimes N) \downarrow_{S}^{A}=M \odot N \downarrow_{S}^{A}
$$

Then one has the following relations:

Lemma 2.5.7. For any $S$-module $V$ and any $A$-module $M$ it follows that 
(1)

$$
\mathcal{V}^{n}(M)=M \otimes\left(\epsilon_{S} \uparrow_{S}^{A}\right)^{n}
$$

(2)

$$
\mathcal{T}^{n+1}(V)=V \uparrow_{S}^{A} \odot \mathcal{T}^{n}\left(\epsilon_{S}\right)
$$

for all $n \geq 1$.

Proof. The first statement follows from Corollary 2.5.2. The second statement is easily proven by induction on $n$.

In the next proposition, we need the Frobenius-Perron theory on Grothendieck rings of hopf algebras developed in [8].

Proposition 2.5.8. Let $A$ be a finite-dimensional Hopf algebra and $L:=\operatorname{LKer}_{M}$ be the kernel of a finite-dimensional $A$-module. Then $\epsilon_{L} \uparrow_{L}^{A}$ is the regular character of $A / / L$.

Proof. For any left $A / / L$-module one has that $N \downarrow_{L}^{A}=|N| \epsilon_{L}$. Applying previous lemma it follows that $N \otimes \epsilon_{L} \uparrow_{L}^{A}=|N| \epsilon_{L} \uparrow_{L}^{A}$. Thus $\epsilon_{L} \uparrow_{L}^{A}$ is the common FrobeniusPerron eigenvector of the operators of left multiplication by all $A / / L$-modules, thus the regular character of $A / / L$ (see [8]).

3. Depth two coideal subalgebras. In this section, we work over a commutative ring $R$. Recall that an extension of $R$-algebras $B \subset A$ is called right (left) depth two if the module $A \otimes_{B} A$ is a direct summand in $A^{n}$ in the category ${ }_{B} \operatorname{Mod}_{A}$ (respectively, ${ }_{A} \operatorname{Mod}_{B}$ ) for an arbitrary $n \geq 1$.

In this section, we will show that a left coideal subalgebra of a Hopf algebra $A$ is right depth two if and only if it is left normal, i.e. closed under the left adjoint action. For this reason, in this section, we have to work with right modules instead of left. Our treatment is very similar to the one used in [4] for depth two Hopf subalgebras.

\subsection{Depth two coideal subalgebras.}

Proposition 3.1.1. Let $S$ be a right coideal subalgebra of a Hopf algebra A.

(1) Then the map

$$
\beta: A \otimes_{S} A \rightarrow A \otimes A / S^{+} A \text { given by } a \otimes_{S} b \mapsto a b_{1} \otimes \overline{b_{2}}
$$

is a well-defined morphism of $(A, S)$-bimodules. The $(A, S)$-bimodule actions are defined as $c\left(a \otimes_{S} b\right) x=c a \otimes b x$ on $A \otimes_{S} A$ and $c(a \otimes \bar{b}) x=c a x \otimes b$ for $a, b, c \in A$ and $x \in S$.

(2) If $A S^{+} \subset S^{+} A$ then the above map $\beta$ is an isomorphism.

Proof. The first item follows by direct computation. For the second item consider

$$
\gamma: A \otimes A / S^{+} A \rightarrow A \otimes_{S} A \text { given by } a \otimes_{S} \bar{b} \mapsto a b_{1} \otimes_{S} S b_{2} .
$$

It is not hard to check that if $A S^{+} \subset S^{+} A$ then $\gamma$ is well defined. Moreover $\gamma$ is an inverse for $\beta$. 
For the rest of this section let $\bar{A}:=A / A S^{+} A$ and $\pi: A \rightarrow \bar{A}$ be the canonical projection. Recall that the extension $A / S$ is $\bar{A}$-right Hopf Galois [11] if $A^{\text {co } \pi}=S$ and the canonical map $\beta$ is bijective.

Proposition 3.1.2. If $A$ is left or right faithfully flat over $S$ and $A S^{+} \subset S A^{+}$then $A / S$ is a right $\bar{A}$-Galois extension. In particular, $S$ is a left normal coideal subalgebra.

Proof. By Proposition 3.1.1, one knows that $\beta$ is bijective. If $A$ is left faithfully flat over $S$ the second theorem from section 13.1 of [17] implies that $S$ equals the equaliser of $i_{1}$ and $i_{2}$ where $i_{1}: A \rightarrow A \otimes_{S} A a \mapsto a \otimes_{S} 1$ and $i_{2}: A \rightarrow A \otimes_{S} A a \mapsto 1 \otimes_{S} a$. Since $\beta$ is bijective, this coincides with the equaliser of $i_{1} \circ \beta$ and $i_{2} \circ \beta$. But this last equaliser is exactly $A^{c o} \pi$. Thus $S$ is also closed under the left adjoint action.

If $A$ is right faithfully flat over $S$ a 'right version' of the same second theorem from Section 13.1 of [17] would also imply that $S$ is the equaliser $i_{1}$ and $i_{2}$.

THEOREM 3.1.3. If $A S^{+} \subset S A^{+}$and $A$ is finitely generated projective as left $S$-module then $S$ is of right depth two inside $A$.

Proof. Using Lemma 2.7 from [4] it follows that $\bar{A} \mid R^{n}$ in $\operatorname{Mod}_{R}$ and therefore $A \otimes_{S} A \cong A \otimes \bar{A} \mid A \otimes R^{n} \cong A^{n}$ in ${ }_{A} \operatorname{Mod}_{S}$.

Proposition 3.1.4. Let $S$ be a left coideal subalgebra of $A$ such that $A$ is faithfully flat $S$-module. If $S$ has right depth two inside $A$ then $A S^{+} \subset S^{+} A$.

Proof. If $S$ has right depth two inside $A$ then $k \otimes_{A}\left(A \otimes_{S} A\right)$ is a direct summand in $\left(k \otimes_{A} A\right)^{n}$ in $\operatorname{Mod}_{S}$. Note that $k \otimes_{S} A$ is the trivial $S$-modules and therefore $k \otimes_{S} A$ divides $k^{n}$ in $S$-mod. Since $S^{+}$annihilates the $S$-module $k$, this implies that the twosided ideal generated $A S^{+} A$ annihilates the $A$-module $k \otimes_{S} A$. Previous remark implies that $A S^{+} A=S^{+} A$ and in particular $A S^{+} \subset S^{+} A$.

COROLlARY 3.1.5. Let $S$ be a coideal subalgebra of $A$ such that $A$ is faithfully flat over $S$. Then $S$ is a right depth two subalgebra of $A$ if and only if $S$ is normal, i.e. closed under the left adjoint action.

3.2. Rieffel's normality for coideal subalgebras. Let $B \subset A$ an extension of finitedimensional $k$-algebras. An ideal $J$ of $B$ is called $A$-invariant if $A J=J A$. Following [14] the extension $A / B$ is called normal if for every maximal two-sided ideal $I$ of $A$ the ideal $B \cap I$ is $A$-invariant.

If $S$ is closed under adjoint action and $A$ has bijective antipode then it easy to verify that $S$ is normal in Rieffel's sense. Indeed, if $I$ is a two-sided ideal of $A$ and $x \in S \cap I$ then $a x=a_{1} x S\left(a_{2}\right) a_{3} \in(I \cap S) A$. Also $x S a=a_{3} S a_{2} x S a_{1} \in A(I \cap S)$. Conversely, if a coideal subalgebra $S$ of a semisimple Hopf algebra $A$ is normal in Rieffel's sense then $A S^{+}=S^{+} A$ since $S^{+}=A^{+} \cap S$ is a maximal two-sided ideal of $S$. Then Proposition 3.1.2 implies that $S$ is closed under the left adjoint action. Thus normality, depth two and Rieffel's normality coincide for coideal subalgebras of a Hopf algebra with bijective antipode.

REMARK 3.2.1. If $A$ is semisimple it will be shown in the next section that $S$ is also semisimple. In this case, left depth two coincides with right depth two by Theorem 4.6 of [6]. 
This remark brings up the question whether left and right depth two coincide on coideal subalgebras. Further, one can ask whether the extension $A / S$ is a quasiFrobenius extension, at least in the finite-dimensional case. Results from [15] shows that $S$ is a Frobenius algebra if $S$ is finite dimensional.

4. The semisimple case. Throughout of this section, we assume that the Hopf algebra $A$ is semisimple. Let $S$ be a left coideal subalgebra of $A$.

Since $A$ is free over $S$ [15] there is a decomposition $A=S \oplus R$ as left $S$-modules. Consider $\Lambda_{A}=x+r$ the decomposition of the idempotent integral $\Lambda_{A}$ in the above direct sum. Then, clearly, $s x=\epsilon(s) x$ for all $s \in S$ and $\epsilon(x)=1$. Similarly, since $A$ is free as right $S$ there is a decomposition $A=S \oplus R^{\prime}$ as left $S$-module. Consider $\Lambda_{A}=y+r^{\prime}$ the decomposition of the idempotent integral $\Lambda_{A}$ in the above direct sum. Then, clearly, $y s=\epsilon(s) x$ for all $s \in S$ and $\epsilon(y)=1$. Thus $x=y x=y$.

LEMMA 4.0.2. Let $S$ be a left coideal subalgebra of a semisimple Hopf algebra A. Then $S$ is also semisimple.

Proof. We will use a Maschke-type argument for the category of $S$-modules. Suppose that $W$ is an $S$ - submodule of $V$ and $f: V \rightarrow W$ a $k$-linear projection. Then it easy to check $\tilde{f}: V \rightarrow W$ given by

$$
\tilde{f}(v)=\sum S x_{1} f\left(x_{2} v\right)
$$

is an $S$-projection. Indeed, one has that $\tilde{f}(s v)=\sum S x_{1} f\left(x_{2} s v\right)=s_{1} S\left(s_{2}\right) S x_{1} f\left(x_{2} s_{3} v\right)=$ $s \tilde{f}(v)$ and $\tilde{f}(w)=S x_{1} x_{2} w=w$.

Thus the element $x$ from above is the central idempotent corresponding to the trivial module $\epsilon_{S}$ of $S$.

4.1. Rieffel's equivalence relation for a coideal subalgebra. Let $S \subseteq A$ be a left coideal subalgebra of $A$.

Let $V$ and $W$ two $S$-modules. that We say that $V \sim W$ if and only if there is a simple $A$-module $M$ such that $V$ and $W$ are both constituents of $M \downarrow_{S}^{A}$. The relation $\sim$ is reflexive and symmetric but not transitive in general. Its transitive closure is denoted by $\approx$. Thus we say that $V \approx W$ if and only if there is $m \geq 1$ and a sequence $V_{i_{0}}, V_{i_{1}}, \ldots, V_{i_{m-1}}, V_{i_{m}}$ of simple $S$-modules such that $V=V_{i_{0}} \sim V_{i_{1}} \sim V_{i_{2}} \sim \ldots \sim$ $V_{i_{m-1}} \sim V_{i_{m}}=W$. As explained in [6], it follows that $V \approx W$ if and only if there is $n \geq 0$ such that $V$ is a constituent to $\mathcal{T}^{n}(W)$. This is equivalent to $W$ to be a constituent of $\mathcal{T}^{n}(V)$.

We denote the above equivalence relation by $d_{S}^{A}$. This equivalence relation is considered in [14] in the context of any extension of semisimple Hopf algebras.

Similarly, one can define an equivalence relation $u_{B}^{A}$ on the set of irreducible $A$ modules. We say that $M \sim N$ for two simple $A$-modules $M$ and $N$ if and only if their restriction to $S$ have a common constituent. Then $u_{S}^{A}$ is the transitive closure of $\sim$. Similarly, $M$ and $N$ are equivalent if and only if there is $n \geq 0$ such that $M$ is a constituent to $\mathcal{V}^{n}(N)$. This is equivalent to $N$ to be a constituent of $\mathcal{V}^{n}(M)$.

4.2. Tensor powers of a character. The following theorem can be viewed as a generalisation of Brauer's theorem for groups: 
THEOREM 4.2.1. Let $A$ be a semisimple Hopf algebra and $M$ be an A-module with character $\chi$. If $L:=\mathrm{LKer}_{M}$ then the irreducible modules of $A / / L$ are precisely all the irreducible constituents the tensor powers $M^{\otimes n}$ with $n \geq 0$.

Proof. One has that $A / / L$ is a semisimple Hopf algebra. On the other hand, since $L$ is left normal Corollary 2.4.3 implies that the simple $A$-submodules of $k \uparrow_{L}^{A}$ and the simple $A$-submodules of $A / / L$ coincide. Then description of the Hopf ideal $I_{M}$ given in Corollary 2.3.7 implies the conclusion.

The next corollary follows from Proposition 3.3 from [5].

Corollary 4.2.2. Let $A$ be a semisimple Hopf algebra and $M$ an $A$ module with character $\chi$. If $\chi$ is central in $A^{*}$ then $\operatorname{Lker}_{\chi}=\operatorname{Rker}_{\chi}=A_{\chi}$.

Proof. Let $L:=$ Lker $_{x}$. Then from the previous theorem and Proposition 3.3 of [5] the quotient Hopf algebras $A / / L$ and $A / / A_{\chi}$ have the same irreducible representations, namely the irreducible representations of all tensor powers of $M$. It follows that $\operatorname{dim}_{k} L=\operatorname{dim}_{k} A_{\chi}$. Since $A_{\chi} \subset L$ then one has $A_{\chi}=L$. Then $A_{\chi}=$ Rker ${ }_{\chi}$ by applying the antipode $S$.

In view of the last corollary, the next theorem can be viewed as an extension of Corollary 6.5 for group algebras from [6].

THEOREM 4.2.3. Let $A$ be a semisimple Hopf algebra and $S$ be a coideal subalgebra of $A$. Let $L:=\operatorname{core}(S)$. Then the equivalence relations $u_{S}^{A}$ and $u_{L}^{A}$ coincide.

Proof. From Proposition 2.4.2 it follows that $L:=\operatorname{LKer}_{\epsilon_{S} \uparrow S}$. Two $A$-modules $M$ and $N$ are equivalent if and only if $N$ is a constituent of $\mathcal{V}^{n}(M)$ for some $n \geq 1$. From the formula of $\mathcal{V}^{n}(M)$ it follows that $M$ and $N$ are equivalent if and only if $N$ is a constituent of $M \otimes\left(\epsilon_{S} \uparrow{ }_{S}^{A}\right)^{n}$ for some $n \geq 1$.

But from Theorem 4.2.1 it follows that $\epsilon_{L} \uparrow_{L}^{A}$ has as constituents all the irreducible constituents of all tensor powers $\left(\epsilon_{S} \uparrow_{S}^{A}\right)^{n}$ with $n \geq 0$. Thus two $A$-modules $M$ and $N$ are equivalent relative to $S$ if and only if they are equivalent relative to $L$.

4.3. The integral element of $S$. Let $x_{S}:=x$ denote the element from the beginning of this section.

In this situation, one has $A S^{+}=A\left(1-x_{S}\right)$. Indeed, if $s \in S^{+}$then $s=s x_{S}+s(1-$ $\left.x_{S}\right)=\epsilon(s) x_{S}+s\left(1-x_{S}\right)=s\left(1-x_{S}\right)$.

Proposition 4.3.1. Let A be a semisimple Hopf algebra and $S$ be a coideal subalgebra of $A$. Then the following statements are equivalent:

(1) $S$ is normal in $A$

(2) $\epsilon_{s}$ by itself from an equivalence class of $d_{s}^{A}$.

(3) The element $x_{S}$ is central in $A$.

Proof. Corollary 2.4.3 implies the equivalence between the first two items. Proposition 3.1 from [6] shows the equivalence between the last two items. From the results from [16] it follows that $S$ is normal if and only if $A S^{+}=S^{+} A$.

Note that the equivalence between first and third item generalises Proposition 1 from [10]. 
ACKNOWLEDGEMENTS. This work was supported by the strategic grant POSDRU/89/1.5/S/58852, project 'Postdoctoral programme for training scientific researchers' cofinanced by the European Social Found within the Sectorial Operational Program Human Resources Development 2007-2013.

\section{REFERENCES}

1. N. Andruskiewitsch, Extensions of Hopf algebras, Can. J. Math. 48(1) (1996), 3-42.

2. N. Andruskiewitsch and J. Devoto, Extensions of Hopf algebras, Algebra $i$ Analiz 7 (1995), 22-69.

3. T. Banica and J. Bichon, Hopf images and inner faithful representations, Glasgow Math. J. 52 (2010), 677-703.

4. R. Boltje and B. Kuelshammer, On the depth 2 condition for group algebra and Hopf algebra extensions, J. Algebra 323(6) (2009), 1783-1796.

5. S. Burciu, Normal Hopf subalgebras of semisimple Hopf algebras, Proc. Amer. Math. Soc. 137(12) (2009), 3969-3979.

6. S. Burciu, L. Kadison and B. Kuelshammer, On subgroup depth, Int. Electron. J. Algebra 9 (2011), 133-166.

7. P. Etingof and S. Gelaki, On the exponent of finite dimensional Hopf Algebras, Math. Res. Lett. 6(2) (1999), 131-140.

8. P. Etingof and V. Ostrik, Finite tensor categories, Moscow J. Math. 4(3) (2004), 627-654.

9. I. M. Isaacs, Character theory of finite groups, in Pure and Applied Mathematics, Vol. 69 (Academic Press, New York/London, 1976).

10. A. Masuoka, Semisimple Hopf algebras of dimension 2p, Commun. Algebra 23(5) (1995), 1931-1940.

11. S. Montgomery, Hopf algebras and their actions on rings, in CBMS regional conference series in mathematics, Vol. 82 (American Mathematical Society, Providence, RI, 1993).

12. D. S. Passman and D. Quinn, Burnside's theorem for Hopf algebras, Proc. Amer. Math. Soc 123 (1995), 327-333.

13. M. Rieffel, Burnside's theorem for representations of Hopf algebras, J. Algebra 6 (1967), $123-130$.

14. M. Rieffel, Normal subrings and induced representations, J. Algebra 24 (1979), 264-386.

15. Y. Skryabin, Projectivity and freeness over comodule algebras, Trans. Amer. Math. Soc 359(6) (2007), 2597-2623. 2523.

16. M. Takeuchi, Quotient spaces for Hopf algebras, Commun. Alg. 22(7) (1995), 2503-

17. W. C. Waterhouse, Introduction to affine group schemes, Vol. 69 (Springer-Verlag, Berlin, 1979). 